\title{
OPEN The relationship of soluble TREM2 to other biomarkers of sporadic Alzheimer's disease
}

\author{
So-Hee Park ${ }^{1,5}$, Eun-Hye Lee ${ }^{2,5}$, Hyung-Ji Kim ${ }^{3}$, Sungyang Jo ${ }^{3}$, Sunju Lee ${ }^{3}$, Sang Won Seo ${ }^{4}$, \\ Hyun-Hee Park ${ }^{2}$, Seong-Ho Koh ${ }^{2 \bowtie}$ \& Jae-Hong Lee ${ }^{3 \bowtie}$
}

Microglial activation is a central player in the pathophysiology of Alzheimer's disease (AD). The soluble fragment of triggering receptor expressed on myeloid cells 2 (sTREM2) can serve as a marker for microglial activation and has been shown to be overexpressed in AD. However, the relationship of STREM2 with other AD biomarkers has not been extensively studied. We investigated the relationship between cerebrospinal fluid (CSF) STREM2 and other AD biomarkers and examined the correlation of plasma sTREM2 with CSF STREM2 in a cohort of individuals with AD and without AD. Participants were consecutively recruited from Asan Medical Center from 2018 to 2020 . Subjects were stratified by their amyloid positivity and clinical status. Along with other AD biomarkers, sTREM2 level was measured in the plasma as well as CSF. In 101 patients with either amyloid-positive or negative status, CSF sTREM2 was closely associated with CSF T-tau and P-tau and not with Abeta42. CSF STREM2 levels were found to be strongly correlated with CSF neurofilament light chain. The comparison of CSF and plasma sTREM2 levels tended to have an inverse correlation. Plasma sTREM2 and P-tau levels were oppositely influenced by age. Our results suggest that neuroinflammation may be closely associated with tauinduced neurodegeneration.

Biomarkers have now become an essential component of Alzheimer's research. Extracellular amyloid plaque and intraneuronal hyperphosphorylated tau accumulation are considered hallmarks of Alzheimer's disease (AD) and many biomarkers have been developed to reflect these central pathophysiological events. By virtue of these biomarkers, we possess a valuable window into the changes occurring in the brain of individuals with Alzheimer's disease and can identify the disease process at the earliest possible stage ${ }^{1}$.

Neuroinflammation has been deemed a secondary phenomenon, but is now emerging as a central player in the development of $\mathrm{AD}^{2-4}$. This theory is centered on microglial activation, which can be brought about by various stimuli, including beta-amyloid. Failure of activated microglia to wall off amyloid plaques from surrounding neurons and phagocytize them leads to the accumulation of amyloid plaques and neurofibrillary tangles ${ }^{5,6}$. Microglial activation is mediated by its membrane-bound receptor, triggering receptor expressed on myeloid cells 2 (TREM2) ${ }^{7}$. The activity of TREM2 can be measured by evaluating the levels of the soluble fragment of TREM2 (sTREM2), which is generated during the TREM2 cleavage process ${ }^{8}$. The concentration of sTREM2 in the cerebrospinal fluid (CSF) has been shown to be elevated in $\mathrm{AD}$, raising the possibility that sTREM2 can serve as a reliable biomarker for $\mathrm{AD}^{9-11}$. It is important to investigate at the relationship of one biomarker with another to better understand the pathophysiology of AD. The role of microglial activation in the amyloid cascade is a matter of curiosity and requires further exploration ${ }^{12}$. However, the relationship between sTREM2 and other conventional biomarkers across the various stages of $\mathrm{AD}$ has not been extensively studied, particularly in patients with sporadic AD. Whether the change in CSF sTREM2 can be reflected in the plasma is also controversial ${ }^{13,14}$. If sTREM2-induced changes in the brain can be reflected in the plasma levels of sTREM2 akin to neurofilament light chain in multiple sclerosis, we will be able to elucidate neuroinflammation associated with AD throughout the course of the disease.

\footnotetext{
${ }^{1}$ Seongnam Center of Senior Health, Seongnam-si, Gyeongi-do 13200, Republic of Korea. ${ }^{2}$ Department of Neurology, Hanyang University Guri Hospital, Hanyang University College of Medicine, Gyeongchun-ro, Guri-Si, Gyeonggi-do 11923, Republic of Korea. ${ }^{3}$ Department of Neurology, University of Ulsan College of Medicine, Asan Medical Center, 88 Olympic-ro 43-gil, Songpa-gu, Seoul 05505, Republic of Korea. ${ }^{4}$ Department of Neurology, Samsung Medical Center, Sungkyunkwan University School of Medicine, Seoul 06351, Republic of Korea. ${ }^{5}$ These authors contributed equally: So-Hee Park and Eun-Hye Lee. ${ }^{\circledR}$ email: ksh213@hanyang.ac.kr; jhlee@amc.seoul.kr
} 


\begin{tabular}{|c|c|c|}
\hline & Amyloid-negative $(\mathrm{N}=47)$ & Amyloid-positive $(\mathrm{N}=54)$ \\
\hline \multicolumn{3}{|l|}{ Population } \\
\hline Sex, n (female) & $47(22)$ & $54(32)$ \\
\hline Age (SD), year ${ }^{*}$ & $71.18(8.66)$ & $66.73(10.36)$ \\
\hline Education level (SD), year & $11.76(4.67)$ & $11.41(4.77)$ \\
\hline Disease duration (SD), months & $28.84(28.12)$ & $35.62(27.10)$ \\
\hline$A P O E \varepsilon 4$ carrier, $\mathrm{n}(\%)^{\star *}$ & $9(18.8)$ & $30(57.7)$ \\
\hline \multicolumn{3}{|l|}{ Underlying disease } \\
\hline $\mathrm{DM}, \mathrm{n}(\%)^{*}$ & $22(44.9)$ & $10(19.2)$ \\
\hline HTN, n (\%) & $22(44.9)$ & $25(48.1)$ \\
\hline Hyperlipidemia, n (\%) & $20(40.8)$ & $26(50.0)$ \\
\hline \multicolumn{3}{|l|}{ Global cognition } \\
\hline $\operatorname{MMSE}(\mathrm{SD})^{*}$ & $24.12(4.65)$ & $22.00(4.90)$ \\
\hline CDR (SD) & $0.67(0.52)$ & $0.72(0.45)$ \\
\hline \multicolumn{3}{|l|}{ Laboratory test } \\
\hline Neutrophil count, \% (SD) & $56.46(8.81)$ & $56.64(8.91)$ \\
\hline Monocyte count, \% (SD) & $8.13(1.61)$ & $11.05(19.01)$ \\
\hline $\mathrm{ESR}(\mathrm{SD}), \mathrm{mm} / \mathrm{h}^{*}$ & $15.47(11.41)$ & $10.91(7.00)$ \\
\hline CRP (SD) & $0.23(0.45)$ & $0.21(0.48)$ \\
\hline $\mathrm{LDL}(\mathrm{SD}), \mathrm{mg} / \mathrm{dL}^{*}$ & $104.78(38.70)$ & $127.03(38.21)$ \\
\hline Albumin (SD), g/dL & $3.69(0.39)$ & $3.84(0.26)$ \\
\hline
\end{tabular}

Table 1. Demographics and clinical characteristics of amyloid-negative and amyloid-positive groups. Demographics and characteristics of the dataset. Student's $t$-test was used for analyzing the age, educational level, disease duration, MMSE, CDR, neutrophil, monocyte, ESR, CRP, and LDL levels. $\chi^{2}$ test was used in the analysis of sex distribution, $A P O E \varepsilon 4, \mathrm{DM}, \mathrm{HTN}$, and hyperlipidemia. DM diabetes mellitus, HTN hypertension, ESR erythrocyte sedimentation rate, CRP C-reactive proteins, $L D L$ low-density lipoprotein. ${ }^{\star}$ Significant at $\mathrm{P}<0.05$. ${ }^{*}$ Significant at $\mathrm{P}<0.01$.

The purpose of this study was to investigate the relationship between CSF sTREM2 and other AD biomarkers and to examine the plasma levels of sTREM2 and its correlation with CSF sTREM2 in a cohort population of $\mathrm{AD}$ and non- $\mathrm{AD}$ conditions.

\section{Results}

Demographics. The clinical characteristics of the participants are presented in Table 1. The amyloid-positive and amyloid-negative groups showed a significant difference in age $(P=0.025)$. The mean age of the amyloidpositive group, in which 21 early-onset patients are included, was lower than that of the amyloid-negative group (66.7 vs. 71.2 year). The frequency of the $A P O E \varepsilon 4$ allele was significantly higher in the amyloid-positive group than that in the amyloid-negative group $(P=0.003$, hazard ratio $=16.57)$. The frequency of diabetes mellitus was higher in the amyloid-negative group than that in the amyloid-positive group $(\mathrm{P}=0.01)$. The amyloid-positive group had poor performances in K-MMSE compared to those of the amyloid-negative group $(\mathrm{P}=0.029)$. The amyloid-positive group showed significantly higher serum LDL levels $(\mathrm{P}=0.042)$ and albumin $(\mathrm{P}=0.02)$. The amyloid-negative group showed higher serum ESR than did the amyloid-positive group $(\mathrm{P}=0.025)$. Other demographic features, including sex distribution, CRP serum levels, frequency of hypertension (HTN), and hyperlipidemia, did not show significant differences between the two groups.

AD biomarkers in the cohort. The CSF A $\beta_{42}$, T-tau, and P-Tau S199 levels were compared between the two groups (amyloid-positive and amyloid-negative) to demonstrate that the cohort had typical patterns of AD core biomarkers. As expected, the CSF A $\beta_{42}$ levels were significantly lower in the amyloid-positive group than those in the amyloid-negative group $(\mathrm{P}<0.001)$ (Fig. 1a). The CSF P-Tau S199 $(\mathrm{P}<0.001)$ and T-tau $(\mathrm{P}<0.001)$ levels were significantly higher in the amyloid-positive group than those in the amyloid-negative group (Fig. 1b,c).

CSF and plasma sTREM2 levels, CSF and plasma biomarkers. In the whole group, the CSF sTREM2 levels were negatively correlated with the plasma sTREM2 levels (Spearman's rho $[\rho]=-0.202, P=0.043$; $\mathrm{n}=101)$ (Fig. 2a,b). The CSF sTREM2 levels positively correlated with the CSF P-Tau S199 $(\rho=0.296, P=0.003$; $\mathrm{n}=100)$ and T-tau levels $(\rho=0.427, \mathrm{P}<0.001 ; \mathrm{n}=99)$ (Fig. $2 \mathrm{a})$. In contrast, the plasma sTREM2 levels were negatively correlated with the CSF P-tau S199 $(\rho=-0.264, \mathrm{P}=0.008 ; \mathrm{n}=100)$ and T-tau levels $(\rho=-0.248, \mathrm{P}=0.013$; $\mathrm{n}=99)$ (Fig. 2a). Plasma sTREM2 levels were significantly correlated with the CSF NfL levels $(\rho=0.359$, $\mathrm{P}<0.001 ; \mathrm{n}=101$ ) (Fig. 2a). The CSF sTREM2 levels were not significantly correlated with the CSF NfL levels $(\rho=0.085, P=0.401 ; n=101)$. With respect to the age effects, age correlated positively with the plasma sTREM2 
a

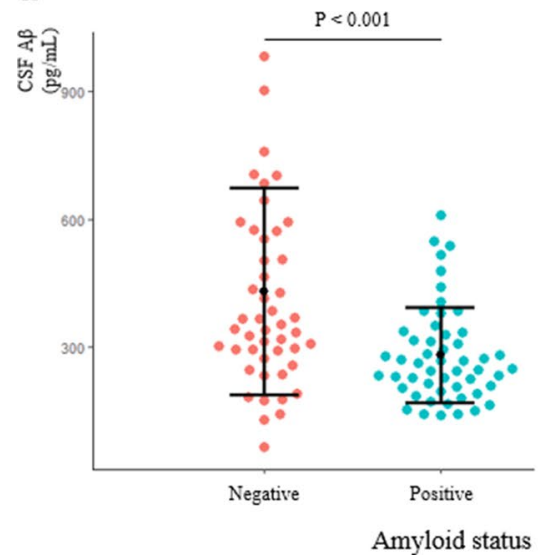

b

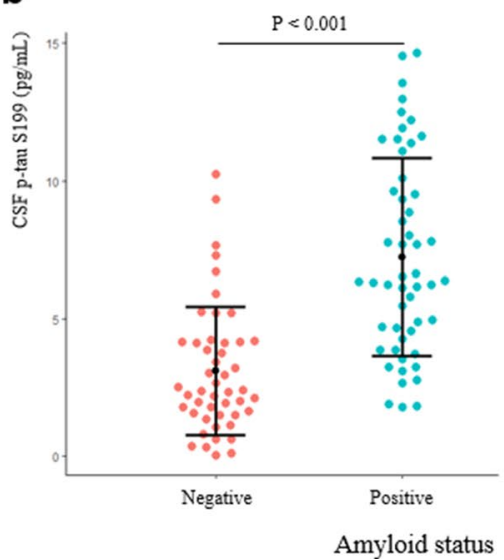

C

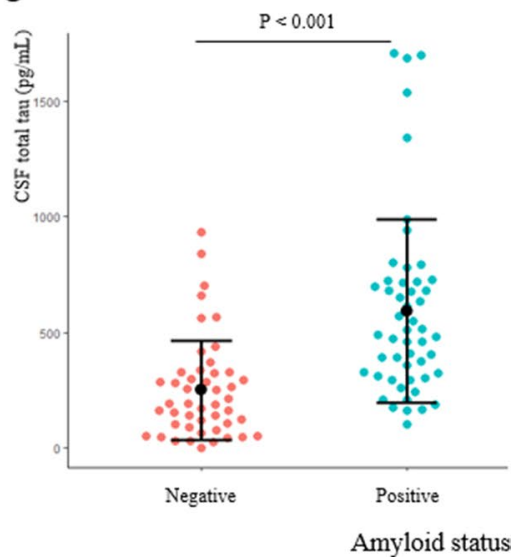

Figure 1. Alzheimer's disease (AD) biomarkers in the cohort. To demonstrate that the cohort had typical AD biomarker patterns, the levels of CSF A $\beta_{42}$, T-tau, and P-tau S199 were compared between the two groups. A $\beta_{42}$ levels were significantly lower in the amyloid-positive group than those in the amyloid-negative group (a). The cerebrospinal fluid (CSF) P-tau S199 (b) and T-tau (c) levels were significantly higher in the amyloid-positive group than those in the amyloid-negative group. Statistical analysis was performed using the Mann-Whitney test. sTREM2 soluble triggering-receptor expressed on myeloid cells 2, NfL neurofilament light chain, $N R G N$ neurogranin. Statistical significance ${ }^{\star} \mathrm{P}<0.05,{ }^{*} \mathrm{P}<0.01$.

a

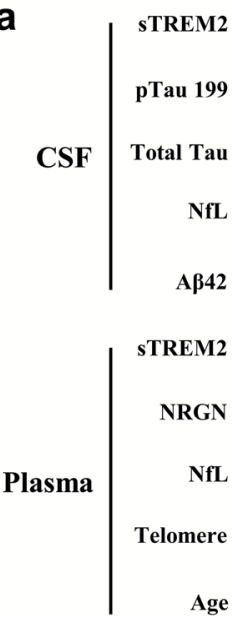

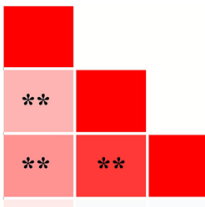

fL
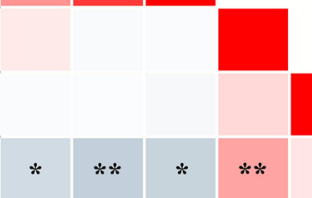

(1)

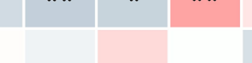

L

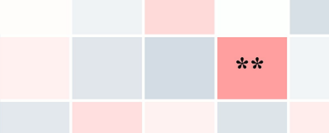

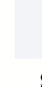

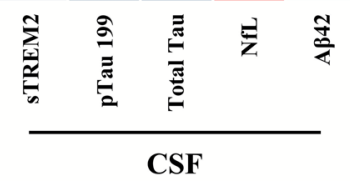

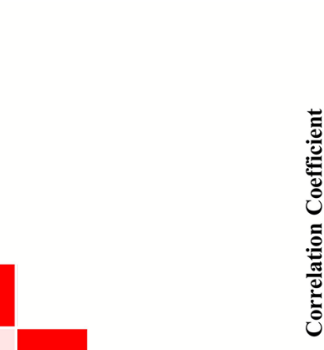

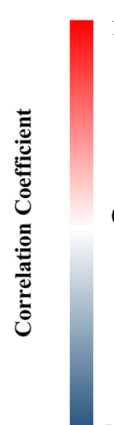

$-1$

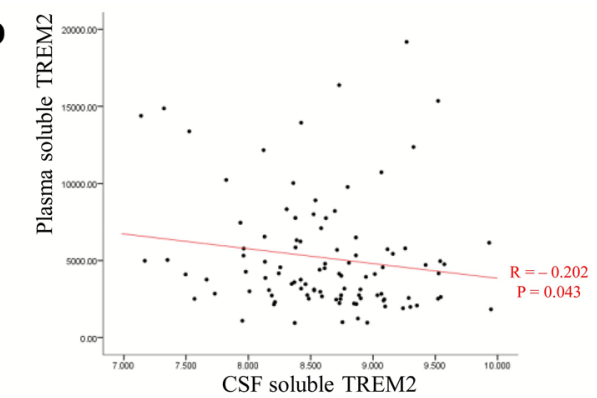

C

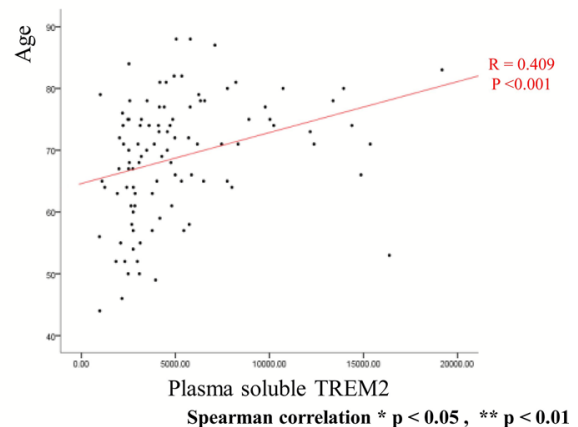

Figure 2. Relationship among biomarkers as a whole dataset. The relationship between cerebrospinal fluid (CSF) and plasma biomarkers is displayed in the heatmap (a). The levels of soluble triggering-receptor expressed on myeloid cells 2 (sTREM2) in CSF and plasma were negatively correlated (b). Age was also positively correlated with the plasma sTREM2 levels (c). Statistical analysis was performed by linear regression analysis. sTREM2 soluble triggering-receptor expressed on myeloid cells 2, NfL neurofilament light chain, NRGN neurogranin. Statistical significance ${ }^{\star} \mathrm{P}<0.05,{ }^{*} \mathrm{P}<0.01$.

levels $(\rho=0.409, \mathrm{P}<0.001 ; \mathrm{n}=101)$ and negatively with the CSF P-tau S199 $(\rho=-0.240, \mathrm{P}=0.016 ; \mathrm{n}=100)$ and T-tau levels $(\rho=-0.258, P=0.010 ; n=99)$ (Fig. $2 c)$.

The plasma sTREM2 levels were positively correlated with the plasma NfL levels $(\rho=0.416, \mathrm{P}<0.001 ; \mathrm{n}=100)$. In contrast, the CSF sTREM2 was not significantly correlated with the plasma biomarkers (Table 2 and Fig. 2a).

Plasma neurodegeneration biomarkers. The plasma sTREM2 positively correlated with plasma NfL $(\rho=0.416, \mathrm{P}<0.001 ; \mathrm{n}=100)$ and age $(\rho=0.409, \mathrm{P}<0.001 ; \mathrm{n}=101)$. Furthermore, NfL and age were strongly correlated with each other $(\rho=0.523, \mathrm{P}<0.001 ; \mathrm{n}=100)$. In contrast, the leukocyte TL was negatively correlated 


\begin{tabular}{|l|l|l|l|l|l|}
\hline \multicolumn{2}{c}{} & \multicolumn{2}{l|}{ CSF sTREM2 } & \multicolumn{2}{l|}{ Plasma sTREM2 } \\
\cline { 3 - 7 } \multicolumn{2}{c|}{} & R & P-value & R & P-value \\
\hline \multirow{4}{*}{ CSF } & A $\beta 42$ & -0.021 & 0.835 & 0.104 & 0.303 \\
\cline { 2 - 6 } & P-tau S199 & 0.296 & 0.003 & -0.264 & 0.008 \\
\cline { 2 - 6 } & T-tau & 0.427 & $<0.001$ & -0.248 & 0.013 \\
\cline { 2 - 6 } & A $\beta 42 /$ P-tau S199 & -0.273 & 0.006 & 0.267 & 0.007 \\
\cline { 2 - 6 } & NfL & 0.085 & 0.401 & 0.359 & $<0.001$ \\
\hline \multirow{4}{*}{ Plasma } & NfL & 0.056 & 0.582 & 0.416 & $<0.001$ \\
\cline { 2 - 6 } & NRGN & 0.012 & 0.906 & -0.068 & 0.512 \\
\cline { 2 - 6 } & Telomere & -0.127 & 0.205 & -0.216 & 0.030 \\
\cline { 2 - 6 } & Age & -0.057 & 0.573 & 0.409 & $<0.001$ \\
\hline
\end{tabular}

Table 2. Correlation of cerebrospinal fluid (CSF) and plasma soluble triggering receptor expressed on myeloid cells 2 (sTREM2) with Alzheimer's disease (AD) biomarkers in all subjects ( $=101)$. Linear regression analyses were used to demonstrate a correlation between biomarkers. NfL neurofilament light chain, NRGN neurogranin.

\begin{tabular}{|l|l|l|l|l|l|}
\hline \multicolumn{2}{c}{} & \multicolumn{2}{l|}{ CSF sTREM2 } & \multicolumn{2}{l|}{ Plasma sTREM2 } \\
\cline { 3 - 6 } \multicolumn{2}{c|}{} & R & P-value & R & P-value \\
\hline \multirow{4}{*}{ CSF } & A $\beta 42$ & -0.152 & 0.281 & 0.128 & 0.365 \\
\cline { 2 - 6 } & P-tau S199 & 0.254 & 0.069 & -0.288 & 0.038 \\
\cline { 2 - 6 } & T-tau & 0.455 & $<0.001$ & -0.227 & 0.110 \\
\cline { 2 - 6 } & A $\beta 42 /$ P-tau S199 & -0.331 & 0.017 & 0.316 & 0.022 \\
\cline { 2 - 6 } & NfL & 0.018 & 0.901 & 0.457 & $<0.001$ \\
\hline \multirow{4}{*}{ Plasma } & NfL & 0.323 & 0.021 & 0.349 & 0.012 \\
\cline { 2 - 6 } & NRGN & 0.080 & 0.584 & 0.126 & 0.389 \\
\cline { 2 - 6 } & Telomere & -0.072 & 0.610 & -0.146 & 0.303 \\
\cline { 2 - 6 } & Age & 0.080 & 0.574 & 0.345 & 0.012 \\
\hline
\end{tabular}

Table 3. Correlation of cerebrospinal fluid (CSF) and plasma soluble triggering receptor expressed on myeloid cells 2 (sTREM2) with Alzheimer's disease (AD) biomarkers in patients with AD continuum (amyloid-positive group) ( $\mathrm{N}=47)$. Linear regression analyses were used to demonstrate a correlation between biomarkers. NfL neurofilament light chain, NRGN neurogranin.

with sTREM2 $(\rho=-0.216, \mathrm{P}<0.03 ; \mathrm{n}=101)$, plasma NfL $(\rho=-0.429, \mathrm{P}<0.001 ; \mathrm{n}=100)$, and age $(\rho=-0.387$, $\mathrm{P}<0.001 ; \mathrm{n}=101$ ), showing that the aging and neurodegeneration factors were progressing together (Fig. 2).

CSF and plasma sTREM2 in the AD continuum. In the amyloid-positive group (AD continuum), the association between CSF and plasma sTREM2 which was observed in the whole dataset disappeared. The CSF sTREM2 levels significantly correlated with CSF T-tau level $(\rho=0.455, P=0.0014 ; n=51)$, and A $\beta 42 / P$-tau S199 $(\rho=-0.331, P=0.017 ; n=52)$. The plasma sTREM2 levels correlated with CSF P-tau S199 $(\rho=-0.288, P=0.038$; $\mathrm{n}=52)$ and $\mathrm{A} \beta_{42} / \mathrm{P}$-tau S199 $(\rho=0.316, \mathrm{P}=0.022 ; \mathrm{n}=52)$, CSF NfL $(\rho=0.457, \mathrm{P}=0.001 ; \mathrm{n}=52)$, and plasma NfL $(\rho=0.349, P=0.012 ; n=51)$. The plasma sTREM2 levels correlated positively with age $(\rho=0.345, P=0.012$; $\mathrm{n}=52$ ) (Table 3 and Fig. $3 a$ ).

CSF and plasma sTREM2 in non-AD condition. In the amyloid-negative conditions, the CSF sTREM2 levels showed a positive correlation with the CSF P-tau S199 $(\rho=0.301, \mathrm{P}=0.038 ; \mathrm{n}=48)$ and T-tau $(\rho=0.425$, $\mathrm{P}=0.003$; $\mathrm{n}=48$ ) levels. No significant significance was found between the plasma sTREM2 and CSF biomarker levels. The plasma sTREM2 levels were correlated with the plasma NfL $(\rho=0.436, P=0.002 ; n=49)$, TL $(\rho=-0.312, P=0.029 ; n=49)$, and age $(\rho=0.430, P=0.002 ; n=49)$ (Table 4 and Fig. $3 b)$.

Relationship of CSF P-tau and T-tau with age stratified by amyloid status. To avoid age bias, all participants were categorized into three different groups, i.e., amyloid-negative, amyloid-positive early-onset (younger than age 65), and amyloid-positive late-onset disease. In case of CSF P-Tau S199, T-tau levels, and 
a

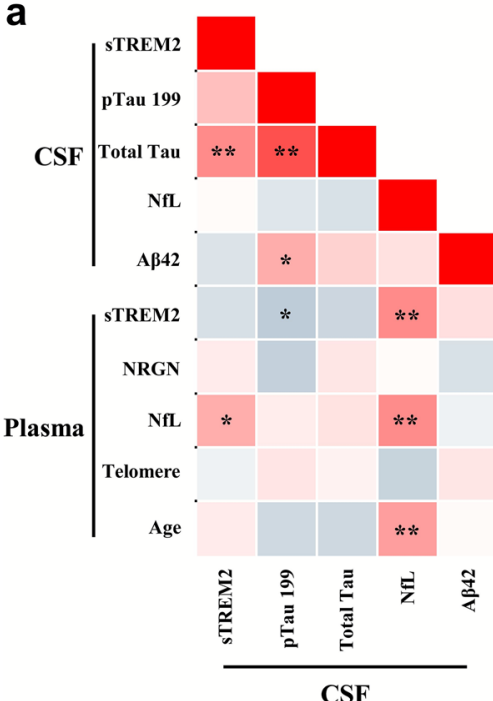

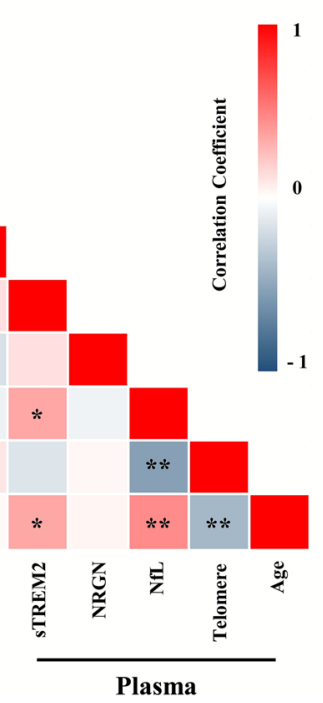

b

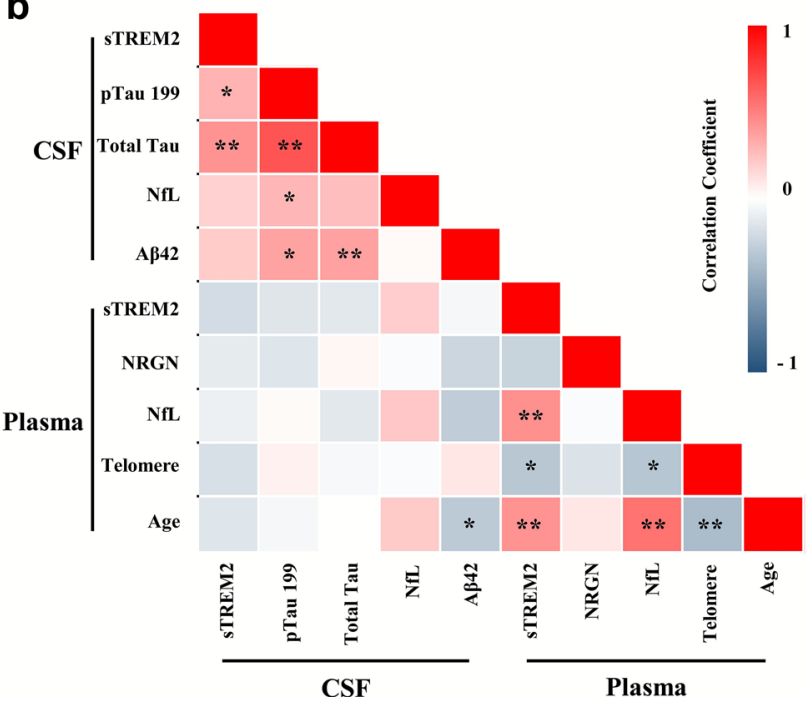

Figure 3. Relationship between biomarkers in the amyloid-positive and -negative groups. The relationship between cerebrospinal fluid (CSF) and plasma biomarker in the amyloid-positive group is displayed in the heatmap (a). The relationship between CSF and plasma biomarker in the amyloid-negative group is displayed in the heatmap (b). Statistical analysis was performed by linear regression analysis. sTREM2 soluble triggeringreceptor expressed on myeloid cells 2, NfL neurofilament light chain, $N R G N$ neurogranin. Statistical significance ${ }^{*} \mathrm{P}<0.05,{ }^{* *} \mathrm{P}<0.01$.

\begin{tabular}{|l|l|l|l|l|l|}
\hline \multicolumn{2}{|c|}{} & \multicolumn{2}{l|}{ CSF sTREM2 } & \multicolumn{2}{l|}{ Plasma sTREM2 } \\
\cline { 3 - 6 } \multicolumn{2}{|c|}{} & R & P-value & R & P-value \\
\hline \multirow{4}{*}{ CSF } & A $\beta 42$ & 0.204 & 0.159 & -0.041 & 0.779 \\
\cline { 2 - 6 } & P-tau S199 & 0.301 & 0.038 & -0.142 & 0.335 \\
\cline { 2 - 6 } & T-tau & 0.425 & 0.003 & -0.126 & 0.394 \\
\cline { 2 - 6 } & A $\beta 42 /$ P-tau S199 & -0.178 & 0.225 & 0.115 & 0.438 \\
\cline { 2 - 6 } & NfL & 0.183 & 0.208 & 0.196 & 0.177 \\
\hline \multirow{4}{*}{ Plasma } & NfL & -0.083 & 0.572 & 0.436 & 0.002 \\
\cline { 2 - 6 } & NRGN & -0.109 & 0.465 & -0.246 & 0.095 \\
\cline { 2 - 6 } & Telomere & -0.175 & 0.230 & -0.312 & 0.029 \\
\cline { 2 - 6 } & Age & -0.140 & 0.338 & 0.430 & 0.002 \\
\hline
\end{tabular}

Table 4. Correlation of cerebrospinal fluid (CSF) and plasma soluble triggering receptor expressed on myeloid cells 2 (sTREM2) with Alzheimer's disease (AD) biomarkers in the amyloid-negative group ( $N=54)$. Linear regression analyses were used to demonstrate a correlation between biomarkers. $N f L$ neurofilament light chain, NRGN neurogranin.

age correlation, we found that the CSF P-tau S199 and T-tau levels were inversely influenced by age in the whole dataset, as previously noted (Fig. 3). The tau pathology over the age course was negatively correlated with CSF P-tau S199 only in the early-onset amyloid-positive group, but this was not significant (Fig. 4a,b). In case of plasma sTREM2, the amyloid-negative group still showed a strong positive correlation with age $(\rho=0.430$, $\mathrm{P}=0.002$ ). However, no significance was observed in the amyloid-positive early or late onset group (Fig. $4 \mathrm{c}$ ).

\section{Discussion}

We collected both plasma and CSF samples from participants in this AD biomarker study to examine whether CSF sTREM2 can be mapped onto other AD biomarkers and also correlate with plasma sTREM2. The major findings of this study were as follows: first, we observed that CSF sTREM2 was closely associated with CSF T-tau and P-tau, but not so much with $\mathrm{A} \beta_{42}$; second, the CSF sTREM2 levels were found to be strongly correlated with CSF NfL; third, comparison of the CSF sTREM2 and plasma sTREM2 levels tended to show an inverse correlation; fourth, the plasma sTREM2 and P-tau levels were influenced by age in an opposite way (higher levels of sTREM2 and lower levels of P-tau were associated with older age). Taken together, these results suggest that 
a

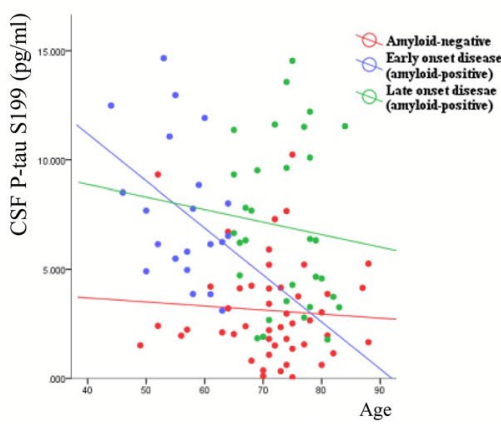

b

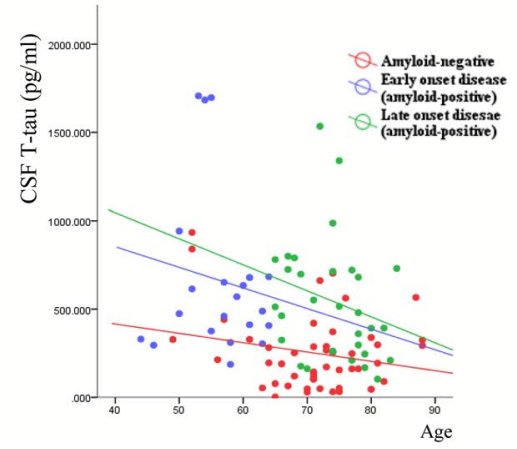

c

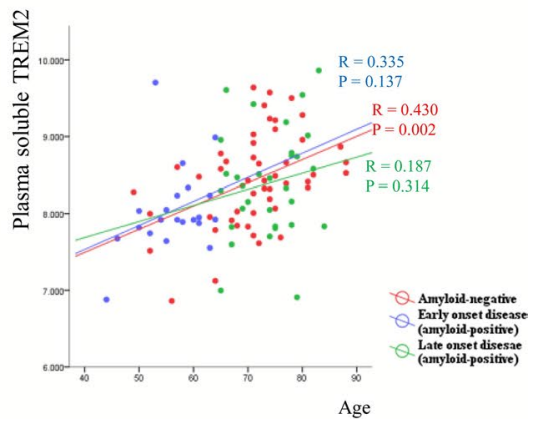

Figure 4. Effect of age on biomarkers. All participants were categorized into three groups: amyloid-negative, amyloid-positive early-onset (younger than age 65), and amyloid-positive late-onset. The tau pathology over the age course was negatively correlated with CSF P-tau S199 only in the early-onset amyloid-positive group, but this was not significant $(\mathbf{a}, \mathbf{b})$. In the case of plasma sTREM2, the amyloid-negative group still showed a strong positive correlation with age (c). Statistical analysis was performed by linear regression analysis.

sTREM2 can serve as a reliable biomarker of $\mathrm{AD}$ and that neuroinflammation may be closely associated with tau-induced neurodegeneration.

With regard to the relationship of sTREM2 to well-known AD biomarkers, a longitudinal study of the DIAN cohort revealed that CSF sTREM2 levels were higher in patients with MCI due to AD than in AD and control groups 9 . Their levels were closely associated with P-tau levels but not with A $\beta_{42}$ levels. The role of sTREM2 in $\mathrm{AD}$ is contentious $\mathrm{s}^{2,6,15-17}$. It could be either a byproduct of full-length TREM2 shedding or an active peptide playing a role in microglial modulation ${ }^{18,19}$. The increase in CSF sTREM2 levels in the early symptomatic stage of $\mathrm{AD}$ was interpreted to reflect a corresponding change in status of microglial activation in response to neurodegeneration induced by hyperphosphorylated tau aggregation ${ }^{8}$. Another cross-sectional study of sporadic AD patients showed that the levels of sTREM2 peaked at the early symptomatic phase of the disease and that the CSF sTREM2 levels were positively correlated with the concentrations of T-tau and P-tau in the CSF, suggesting the important role of sTREM2 in the development of AD pathology and neurodegeneration ${ }^{9,20,21}$. We can suggest that elevated levels of sTREM2 may have close relationship to inducing tau phosphorylation in neurites neighboring amyloid plaques ${ }^{22,23}$. Microglial clustering around amyloid plaques represented by increased sTREM2 levels play a role in initially providing a barrier function ${ }^{24}$. If it fails to wall off amyloid plaques from surrounding neurons and phagocytize them, this can lead to the propagation of amyloid plaques and formation of neurofibrillary tangles ${ }^{25,26}$. Dystrophic neurites containing hyperphosphorylated tau can be found around amyloid plaques during this process ${ }^{27}$. Why CSF sTREM2 was not significantly associated with $A \beta_{42}$ in our sample is unknown. Given the early appearance of $A \beta_{42}$ and its plateau in the prodromal stage of $A D$, giving way to the increase in P-tau, sTREM2 may be closely linked with A $\beta$-triggered tau phosphorylation occurring after $\mathrm{A} \beta_{42}$ accumulation ${ }^{11,28}$. Tau aggregations might affect the microglial activation state, suppressing $A \beta$ clearance and producing neurodegeneration ${ }^{29,30}$. sTREM2 could serve as a marker for the tau-dependent pathogenetic pathway of $\mathrm{AD}^{31,32}$.

Plasma sTREM2 levels were found to be strongly correlated with CSF neurofilament light chain (NfL). These results are in accordance with those of prior studies ${ }^{33}$. Increased levels of NfL are thought to represent axonal damage induced by neuroinflammatory conditions, typically exemplified by multiple sclerosis ${ }^{34}$. These two proteins moving in the same direction indicate that sTREM2 can reflect microglial activation and consequent neuroinflammation. The comparison of CSF sTREM2 and plasma sTREM2 levels revealed no definite relationship with AD continuum. The CSF sTREM2 levels showed a trend toward having an inverse correlation with the plasma sTREM2 levels. This disconnect between CSF sTREM2 and plasma sTREM2 is somewhat difficult to explain. In multiple sclerosis, there is a strong positive correlation between CSF NfL and plasma NfL, indicating the same direction for peripheral and central neuroinflammation ${ }^{13,35}$. In $\mathrm{AD}$, however, the relationship between CSF sTREM2 and plasma STREM2 is not straightforward, and previous reports on this are inconsistent ${ }^{36-38}$. One study performed on AD showed a significant association between peripheral sTREM2 and CSF sTREM2 levels ${ }^{13}$. The opposite results were reported in a study performed on AD and healthy subjects, where increased levels of CSF sTREM2 were observed in AD patients, particularly in the early stage; however, plasma levels were not different between the two groups, suggesting incorrect plasma sTREM2 levels ${ }^{11,39}$. In AD, the plasma sTREM2 levels may not necessarily reflect the CSF sTREM2 levels, and may not follow the same dynamics as CSF STREM $2^{33,40}$. Further studies are required to clarify this.

It is well known that there is an age-related increment in tau pathology, as with the increasing production of tau protein in the brain of aging individuals ${ }^{41-43}$, however the different way that age impacts the CSF sTREM2 and tau levels is intriguing ${ }^{44,45}$. Higher levels of plasma sTREM2 were associated with older age, whereas lower levels of tau were correlated with older age. Aging per se is represented by a chronic and systemic low-grade inflammatory process. In this context, increased levels of plasma STREM2, a potential neuroinflammatory biomarker, are not surprising in older subjects, but decreased levels of CSF P-tau and T-tau in the elderly are quite puzzling, even more so given the apparent positive correlation between the CSF sTREM 2 and tau levels. These are the findings derived from the group as a whole across the amyloid and clinical status. When we focused on 
the $\mathrm{AD}$ continuum, however, the age effect on the CSF tau levels disappeared ${ }^{46}$. As we broke down the subjects into earlier onset ( $<65$ years) and late onset $\mathrm{AD}$ (Fig. 4), we found that this inverse relationship was primarily driven by the earlier onset $\mathrm{AD}$ patients. It is well known that EOAD has higher AD pathology burden including P-tau ${ }^{47-51}$. Since a significant proportion of earlier onset AD subjects happened to be enrolled into our study, this age disproportion was likely to have affected the results, giving rise to the spurious finding.

Our study has several limitations. First, this is a single-center prospective study; thus, sampling bias might exist. Second, with many groups present based on the amyloid status and cognitive severity, the sample size of each group is not large enough to show sufficient statistical power; the validation of our findings through a large-scale study is required. Third, the data on biomarkers in this study are from the baseline of this cohort and longitudinal changes in STREM2 are not available. It would be more interesting if we continued to collect followup data on biomarkers in this cohort to see how the sTREM2 levels fluctuate over the course of AD. Fourth, we chose P-tau S199 for tau hyperphosphorylation status over P-tau181 or P-tau217 either of which is generally preferred for phosphorylated tau measurement. Prior to this study, we verified that P-tauS199 was comparable to the other epitopes through a literature search ${ }^{52,53}$. Fifth, we did not incorporate the apolipoprotein E genotype into the relationship between sTREM2 and other biomarkers, which needs to be further explored ${ }^{54,55}$.

However, despite these limitations, to the best of our knowledge, to date, this is the first attempt to explore the importance of sTREM2 in relation to other AD biomarkers using both CSF and plasma in individuals with and without $\mathrm{AD}$ pathology. We are planning to analyze forthcoming longitudinal data using more competitive markers such as $A \beta_{42} / A \beta_{40}$ ratio, P-tau 181 and P-tau 231 in order to solidify our data.

\section{Conclusion}

The results of this study suggest that soluble TREM2 may serve as a potentially useful biomarker for the microglial status and consequent neuroinflammation in the AD continuum. Thus, great emphasis should be placed on sTREM2 so as to obtain a more complete picture of AD.

\section{Methods}

Participants. We enrolled 104 participants prospectively from the memory clinic of Asan Medical Center, Seoul, Korea from June 2018 to July 2020. All participants or their proxies signed an informed consent form. All subjects underwent brain MRI, comprehensive neuropsychological testing, fluorine-18 $\left[{ }^{18} \mathrm{~F}\right]$-florbetaben amyloid positron emission tomography (PET), and CSF analysis. Subjects were included based on the following criteria: (1) aged over 40 years and under 90 years; (2) no evidence of parenchymal lesions that could influence the cognitive function based on brain MRI. Three subjects were excluded from the dataset because of the withdrawal of consent and thus a total of 101 subjects were included in this study.

All PET images were obtained using Discovery 690, 710, and 690 Elite PET/computed tomography scanners (GE Healthcare; Chicago, IL, USA). Amyloid PET images were collected for 20 min, beginning 90 min after injection of $300 \pm 30 \mathrm{MBq}{ }^{18} \mathrm{~F}$-florbetaben. Two neurologists (H.J.K and J.H.L) and two nuclear medicine physicians (J.S.K. and M.O.) reviewed the PET scans according to the predefined regional cortical tracer uptake (RCTU) and brain amyloid plaque load (BAPL) scoring system. In general, four regions of interest, comprising the frontal, temporal, and parietal cortex and posterior cingulate/precuneus, were interpreted in the visual assessment of the $\left[{ }^{18} \mathrm{~F}\right]$-florbetaben PET scans. The RCTU scores were then condensed into a single three-grade scoring system for each PET scan (BAPL score): 1 , no $\beta$-amyloid load; 2 , minor $\beta$-amyloid load; 3 , significant $\beta$-amyloid load. The final score was reached by consensus, with a BAPL score of 1 regarded as amyloid negative (A $\beta$-) and BAPL scores of 2 and 3 considered to be amyloid positive $(A \beta+)^{56}$. All participants were classified into one of these two groups according to the PET results.

We divided subjects into amyloid positive and negative groups according to the amyloid status on PET irrespective of the cognitive status. In each group, therefore, there exists a cognitive continuum spanning from SMI to dementia.

All of the participants underwent the following blood tests: complete blood count; lipid profile; erythrocyte sedimentation rate (ESR); vitamin $\mathrm{B}_{12}$, folate, and homocysteine serum level test; and thyroid function test. The apolipoprotein $\mathrm{E}(A P O E)$ genotype was identified after extracting genomic DNA from the venous blood. All CSF samples were subjected to complete cell count, protein, and albumin tests.

This study was approved by the Institutional Review Board of Asan Medical Center, Republic of Korea (\#2018-0614). All methods were carried out in accordance with relevant guidelines and regulations.

Reagents. Proximity ligation assay (PLA) buffer, comprising $1 \mathrm{mM}$ D-biotin (Life Technologies), $0.1 \%$ purified BSA (Bovogen), 0.05\% Tween-20 (Sigma), $100 \mathrm{nM}$ goat IgG (Sigma), $0.1 \mu \mathrm{g} / \mu \mathrm{L}$ salmon sperm DNA (Life Technologies), and $5 \mathrm{mM}$ EDTA in PBS (pH 7.4) (Sigma), was stored at - $20{ }^{\circ} \mathrm{C}$. Conjugate probe sequences were provided as below. Streptavidin conjugated oligonucleotide SLC1 (Streptavidin-5'-CGCATCGCCCTT GGACTACGACTGACGAAC CGCTTTGCCTGACTGATCGCTAAATCGTG-3') and SLC2 (5'-TCGTGT CTAAAGTCC GTTACCTTGATTCCCCTAACCCTCTTGAAAAATTCGGCATCGGTGA-3'-streptavidin) were purchased from Solulink. The ligation was conducted by using polymerase chain reaction (PCR) primer 1 (reverse) 5'-GGGAATCAAGGTAACGGACTTTAG-3', PCR primer 2 (forward) 5'-CATCGCCC TTGGAC TACGA-3, and PCR primer 3 (splint) 5'-TACTTAGACACGACACGATTTAG TTT-3'.

Solid phase proximity ligation assay (spPLA). To detect sTREM2 in the CSF and plasma, a spPLA was conducted following the previous protocol ${ }^{57}$. spPLA is able to detect proteins from 1 or $10 \mathrm{nM}$ to femtomolar concentrations. sTREM2 concentrations in the CSF and plasma were measured using biotinylated anti-sTREM2 polyclonal antibody and recombinant TREM2 protein (R\&D systems). Briefly, $1 \mathrm{mg} / \mathrm{mL}$ Dynabeads MyOne 
Streptavidin T1 (Invitrogen) was incubated with $50 \mathrm{nM}$ biotinylated anti-sTREM2 polyclonal antibody (R\&D systems) for $1 \mathrm{~h}$ at room temperature (RT) under rotation to immobilize the sTREM2 antibodies to the magnetic beads. The magnetic beads were washed twice with $0.05 \%$ Tween 20 in 1 X PBS (washing buffer). During the reaction, plasma samples were diluted twofold in PLA buffer, and the recombinant TREM2 protein (R\&D systems) was serially spiked in PLA buffer from $1 \mathrm{nM}$ to $1 \mathrm{fM}$ as a standard. The diluted plasma samples and serial dilution of the standards were then mixed with the antibody-conjugated magnetic beads and incubated for 90 min under rotation. Then, the beads were washed two times with washing buffer. The PLA probes were formed by separately incubating $50 \mathrm{nM}$ of the streptavidin-oligonucleotide conjugates SLC1 and SLC2 with $50 \mathrm{nM}$ biotinylated anti-sTREM2 antibody for $1 \mathrm{~h}$ at RT. Prior to use, the SLC1- and SLC2-anti-sTREM2 antibodies were mixed at an equal ratio and incubated for $5 \mathrm{~min}$ at RT. The final concentration of each probe was $500 \mathrm{pM}$. Finally, the magnetic beads were mixed with $1 \mathrm{nM}$ of PLA probe mix and incubated for another $90 \mathrm{~min}$ at RT followed by washing twice with washing buffer. Real-time PCR mix (1X PCR buffer [QIAGEN] comprising $2.5 \mathrm{mM} \mathrm{MgCl}_{2}$ [QIAGEN], $0.22 \mu \mathrm{M}$ Sybr Green [Life Technologies], $0.1 \mu \mathrm{M}$ of each primer [reverse primer, forward primer, and splint primer] [IDT], $80 \mu \mathrm{M}$ ATP [ThermoFisher], $0.2 \mathrm{mM}$ dNTP with U [ThermoFisher], 0.03 $\mathrm{U} / \mu \mathrm{L}$ Taq polymerase [QIAGEN], $0.01 \mathrm{U} / \mu \mathrm{L}$ T4 DNA ligase [ThermoFisher], $0.02 \mathrm{U} / \mu \mathrm{L}$ UNG [ThermoFisher], and nuclease-free water [QIAGEN]) was prepared as previously described. Before the cycling stage, a heat incubation step was performed for $15 \mathrm{~min}$ at $95^{\circ} \mathrm{C}$, and application steps were performed at 40 cycles for $30 \mathrm{~s}$ at $94{ }^{\circ} \mathrm{C}, 1 \mathrm{~min}$ at $50{ }^{\circ} \mathrm{C}$, and $1 \mathrm{~min}$ at $72{ }^{\circ} \mathrm{C}$. A StepOnePlus real-time PCR instrument (Applied Biosystems) was used for the experiment and analysis. To investigate the performance efficiency of sTREM2 spPLA, LLOD, LLOQ, precision, and dilutional linearity were measured. The LLOD formula was CtLOD $=\mathrm{CtN}-2 \mathrm{SN}$. CtN is the average of $\mathrm{Ct}$ value gained from the background noise and $\mathrm{SN}$ is the standard deviation of this value. sTREM2 spPLA had inter-assay precision $<17.5 \%$ and intra-assay precision $<2 \%$. In terms of inter-assay precision, previous study explained that the relatively large coefficients of variation are the results of the PCR step, which can be highly variable at low target copy numbers. The sTREM2 spPLA showed LLOD 50.58 pg/mL, LLOQ 151.75 pg/ $\mathrm{mL}$ and average $95.9 \%$ dilutional linearity.

Plasma and CSF biomarkers. To measure the levels of the biomarkers, we conducted ELISA and SIMOA following the instructions provided from commercial suppliers. For p-tau S199 measurement, Tau (Phospho) pS199 Human ELISA Kit (Invitrogen) was performed with CSF samples. Briefly, standards and samples were spiked with standard diluent buffer at 1:1 ratio and added 96 wells plate coated with capture antibodies against P-Tau S199 for $2 \mathrm{~h}$. Then, unattached antigens were washed out. The human P-Tau S199 detection antibody solution applied to the wells for $1 \mathrm{~h}$ following $30 \mathrm{~min}$ of incubation with anti-rabbit IgG HRP solution. Finally, the chromogen was added to each well and incubated for $30 \mathrm{~min}$. The reaction was stopped with stop solution and read the absorbance at $450 \mathrm{~nm}$. For total-tau in CSF, human tau (Total) ELISA kit (Invitrogen) was conducted. The standards and samples were diluted with standard diluent buffer at 1:1 ratio and added to the wells coated with capture antibodies for $2 \mathrm{~h}$ at room temperature. After washing, human total tau biotin conjugate antibodies were added to each well for $1 \mathrm{~h}$. Then, streptavidin-HRP solution was applied to the wells for $30 \mathrm{~min}$. Finally, stabilized chromogen and stop solutions were added to each well. The level of total tau in CSF was read at $450 \mathrm{~nm}$. In case of A $\beta 42$ levels, the CSF was diluted threefold with sample diluent due to high endogenous levels. The standards and samples were added and incubated for $2 \mathrm{~h}$ at $2-8^{\circ} \mathrm{C}$. After 4 times of washing, human amyloid $\beta$ (aa1-42) conjugate added to each well and incubated for another $2 \mathrm{~h}$ at at $2-8{ }^{\circ} \mathrm{C}$. The wells were washed out and substrate was applied and reacted with for $30 \mathrm{~min}$ at room temperature. The level of $\mathrm{A} \beta 42$ was determined using a microplate reader (Biotek) set to $450 \mathrm{~nm}$.

For plasma neurogranin, Human Neurogranin ELISA (Lifespan Biosciences) was used. Samples were applied to the wells for $1 \mathrm{~h}$. After washing step, detection reagent $\mathrm{A}$ and $\mathrm{B}$ was added to the wells for $1 \mathrm{~h}$ each at $37^{\circ} \mathrm{C}$. TMB substrate reaction was proceeded for $15 \mathrm{~min}$ and the optical density was determined at $450 \mathrm{~nm}$. Finally, the level of NfL in CSF and plasma was detected using Simoa NF-light Advantage Kit. Plasma and CSF were diluted 4-folds and 100-folds, respectively. CSF and plasma NfL were measured in the DNA Link Laboratory, South Korea, on the Simoa-HD1 platform as previously described ${ }^{57}$.

Telomere length (TL) assay. DNA was extracted from the whole blood using D-DEX IIb RBC Lysis Buffer and D-DEX IIb Cell Lysis Buffer (Intron, MA, USA). DNA hydration was performed with $300 \mu \mathrm{L}$ of DNA hydration solution (QIAGEN, Hilden, Germany). TL analysis was carried out using a nonradioactive TeloTAGGG TL Assay (Roche Boehringer-Mannheim, Grenzach-Wyhlen, Germany) according to the manufacturer's instructions. Approximately 2-4 $\mu$ g of DNA from each sample was digested with Hinf I/RsaI enzyme mix and isolated by gel electrophoresis. DNA fragments were transferred to a nylon membrane (Millipore, Bedford, MA, USA) by Southern transfer and hybridized to digoxigenin (DIG)-labeled probes specific for telomeric repeats. The membrane was incubated with DIG-specific antibodies conjugated to alkaline phosphatase, and the probe was visualized by chemiluminescence detection and an image analyzer (ImageQuant LAS 4000; GE Healthcare, Little Chalfont, UK). The mean telomeric repeat binding factor lengths were determined by comparing them to the molecular weight standards.

Statistical analysis. The statistical analyses included a $\chi^{2}$ test to compare group differences in dichotomous variables, such as sex, diabetes, hypertension, and dyslipidemia, between two groups including the effect of the APOE genotype. To compare the levels of CSF A $\beta_{42}$, T-tau, and P-tau S199 between amyloid positive and negative groups, Mann-Whitney test was used. Linear regression analyses were used to demonstrate a correlation between the CSF and plasma biomarkers. We defined a p-value less than 0.05 was statistically significant (SPSS Version 21.0; IBM Corp., Armonk, NY, USA). 
Received: 26 February 2021; Accepted: 28 May 2021

Published online: 22 June 2021

\section{References}

1. Bjorkli, C., Sandvig, A. \& Sandvig, I. Bridging the gap between fluid biomarkers for Alzheimer's disease, model systems, and patients. Front. Aging Neurosci. 12, 272 (2020).

2. Obulesu, M. \& Jhansilakshmi, M. Neuroinflammation in Alzheimer's disease: An understanding of physiology and pathology. Int. J. Neurosci. 124, 227-235 (2014).

3. Heneka, M. T. et al. Neuroinflammation in Alzheimer's disease. Lancet Neurol. 14, 388-405 (2015).

4. Calsolaro, V. \& Edison, P. Neuroinflammation in Alzheimer's disease: Current evidence and future directions. Alzheimers Dement. 12, 719-732 (2016).

5. Keren-Shaul, H. et al. A unique microglia type associated with restricting development of Alzheimer's disease. Cell 169, 1276-1290 (2017).

6. Hansen, D. V., Hanson, J. E. \& Sheng, M. Microglia in Alzheimer's disease. J. Cell Biol. 217, 459-472 (2018).

7. Yeh, F. L., Hansen, D. V. \& Sheng, M. TREM2, microglia, and neurodegenerative diseases. Trends Mol. Med. 23, 512-533 (2017).

8. Ulrich, J. D., Ulland, T. K., Colonna, M. \& Holtzman, D. M. Elucidating the role of TREM2 in Alzheimer's disease. Neuron 94, 237-248 (2017).

9. Suarez-Calvet, M. et al. Early changes in CSF sTREM2 in dominantly inherited Alzheimer's disease occur after amyloid deposition and neuronal injury. Sci. Transl. Med. 8, 178-369 (2016).

10. Suarez-Calvet, M. et al. sTREM2 cerebrospinal fluid levels are a potential biomarker for microglia activity in early-stage Alzheimer's disease and associate with neuronal injury markers. EMBO Mol. Med. 8, 466-476 (2016).

11. Liu, D. et al. Soluble TREM2 changes during the clinical course of Alzheimer's disease: A meta-analysis. Neurosci. Lett. 686, 10-16 (2018).

12. Ewers, M. et al. Higher CSF sTREM2 and microglia activation are associated with slower rates of beta-amyloid accumulation. EMBO Mol. Med. 12, e12308 (2020).

13. Bekris, L. M. et al. Soluble TREM2 and biomarkers of central and peripheral inflammation in neurodegenerative disease. J. Neuroimmunol. 319, 19-27 (2018).

14. Ohara, T. et al. Serum soluble triggering receptor expressed on myeloid cells 2 as a biomarker for incident dementia: The Hisayama study. Ann. Neurol. 85, 47-58 (2019).

15. Zhong, L. et al. Soluble TREM2 induces inflammatory responses and enhances microglial survival. J. Exp. Med. 214, 597-607 (2017).

16. Heslegrave, A. et al. Increased cerebrospinal fluid soluble TREM2 concentration in Alzheimer's disease. Mol. Neurodegener. 11, 3 (2016).

17. Ewers, M. et al. Increased soluble TREM2 in cerebrospinal fluid is associated with reduced cognitive and clinical decline in Alzheimer's disease. Sci. Transl. Med. 11, 6221 (2019).

18. Yang, J. et al. TREM2 ectodomain and its soluble form in Alzheimer's disease. J. Neuroinflammation 17, 204 (2020).

19. Damisah, E. C., Rai, A. \& Grutzendler, J. TREM2: Modulator of lipid metabolism in microglia. Neuron 105, 759-761 (2020).

20. Singh, A. K. et al. Role of TREM2 in Alzheimer's disease and its consequences on $\beta$-amyloid, tau and neurofibrillary tangles. Curr. Alzheimer Res. 16, 1216-1229 (2019).

21. Deczkowska, A., Weiner, A. \& Amit, I. The physiology, pathology, and potential therapeutic applications of the TREM2 signaling pathway. Cell 181, 1207-1217 (2020).

22. Halaas, N. B. et al. CSF sTREM2 and tau work together in predicting increased temporal lobe atrophy in older adults. Cereb. Cortex 30, 2295-2306 (2020).

23. Wang, Y. et al. TREM2 lipid sensing sustains the microglial response in an Alzheimer's disease model. Cell 160, 1061-1071 (2015).

24. Griciuc, A. et al. TREM2 acts downstream of $\mathrm{CD} 33$ in modulating microglial pathology in Alzheimer's disease. Neuron 103, 820-835 (2019).

25. Yuan, P. et al. TREM2 haplodeficiency in mice and humans impairs the microglia barrier function leading to decreased amyloid compaction and severe axonal dystrophy. Neuron 90, 724-739 (2016).

26. Meilandt, W. J. et al. Trem2 deletion reduces late-stage amyloid plaque accumulation, elevates the $A \beta 42: A \beta 40$ ratio, and exacerbates axonal dystrophy and dendritic spine loss in the PS2APP Alzheimer's mouse model. J. Neurosci. 40, 1956-1974 (2020).

27. Condello, C., Yuan, P. \& Grutzendler, J. Microglia-mediated neuroprotection, TREM2, and Alzheimer's disease: Evidence from optical imaging. Biol. Psychiatry 83, 377-387 (2018).

28. Ma, L. Z. et al. Dynamic changes of CSF sTREM2 in preclinical Alzheimer's disease: The CABLE study. Mol. Neurodegener. 15, 25 (2020).

29. Nisbet, R. M., Polanco, J. C., Ittner, L. M. \& Götz, J. Tau aggregation and its interplay with amyloid- $\beta$. Acta Neuropathol. 129, 207-220 (2015).

30. Xin, S. H., Tan, L., Cao, X., Yu, J. T. \& Tan, L. Clearance of amyloid beta and tau in Alzheimer's disease: From mechanisms to therapy. Neurotox. Res. 34, 733-748 (2018).

31. Jiang, T. et al. TREM2 ameliorates neuronal tau pathology through suppression of microglial inflammatory response. Inflammation 41, 811-823 (2018).

32. Leyns, C. E. G. et al. TREM2 function impedes tau seeding in neuritic plaques. Nat. Neurosci. 22, 1217-1222 (2019).

33. Ashton, N. J. et al. Plasma levels of soluble TREM2 and neurofilament light chain in TREM2 rare variant carriers. Alzheimers Res. Ther. 11, 94 (2019).

34. Kuhle, J. et al. Blood neurofilament light chain as a biomarker of MS disease activity and treatment response. Neurology $\mathbf{9 2}$, e1007-e1015 (2019).

35. Disanto, G. et al. Serum neurofilament light: A biomarker of neuronal damage in multiple sclerosis. Ann. Neurol. 81, 857-870 (2017).

36. Hu, N. et al. Increased expression of TREM2 in peripheral blood of Alzheimer's disease patients. J. Alzheimers Dis. 38, 497-501 (2014).

37. Suárez-Calvet, M. et al. Early increase of CSF sTREM2 in Alzheimer's disease is associated with tau related-neurodegeneration but not with amyloid- $\beta$ pathology. Mol. Neurodegener. 14, 1 (2019).

38. Casati, M. et al. Increased expression of TREM2 in peripheral cells from mild cognitive impairment patients who progress into Alzheimer's disease. Eur J Neurol 25, 805-810 (2018).

39. Piccio, L. et al. Cerebrospinal fluid soluble TREM2 is higher in Alzheimer disease and associated with mutation status. Acta Neuropathol. 131, 925-933 (2016).

40. Weber, G. E. et al. An altered relationship between soluble trem 2 and inflammatory markers in young adults with down syndrome: A preliminary report. J. Immunol. 204, 1111-1118 (2020).

41. Yue, F., Lu, C., Ai, Y., Chan, P. \& Zhang, Z. Age-associated changes of cerebrospinal fluid amyloid- $\beta$ and tau in cynomolgus monkeys. Neurobiol. Aging 35, 1656-1659 (2014). 
42. Bennett, D. A. et al. Neuropathology of older persons without cognitive impairment from two community-based studies. Neurology 66, 1837-1844 (2006).

43. Reas, E. T. Amyloid and tau pathology in normal cognitive aging. J. Neurosci. 37, 7561-7563 (2017).

44. Henjum, K. et al. Cerebrospinal fluid soluble TREM2 in aging and Alzheimer's disease. Alzheimers Res. Ther. 8, 17 (2016).

45. Zhao, N. et al. Alzheimer's risk factors age, APOE genotype, and sex drive distinct molecular pathways. Neuron 106, 727-742 (2020).

46. Sjögren, M. et al. Tau and A $\beta 42$ in cerebrospinal fluid from healthy adults 21-93 years of age: Establishment of reference values. Clin. Chem. 47, 1776-1781 (2001).

47. Koedam, E. L. et al. Early-versus late-onset Alzheimer's disease: More than age alone. J. Alzheimers Dis. 19, 1401-1408 (2010).

48. Marshall, G. A., Fairbanks, L. A., Tekin, S., Vinters, H. V. \& Cummings, J. L. Early-onset Alzheimer's disease is associated with greater pathologic burden. J. Geriatr. Psychiatry Neurol. 20, 29-33 (2007).

49. Lam, B., Masellis, M., Freedman, M., Stuss, D. T. \& Black, S. E. Clinical, imaging, and pathological heterogeneity of the Alzheimer's disease syndrome. Alzheimer's Res. Ther. 5, 1 (2013).

50. Mizuno, Y., Ikeda, K., Tsuchiya, K., Ishihara, R. \& Shibayama, H. Two distinct subgroups of senile dementia of Alzheimer type: Quantitative study of neurofibrillary tangles. Neuropathology 23, 282-289 (2003).

51. Ho, G. J. et al. Age at onset is associated with disease severity in Lewy body variant and Alzheimer's disease. NeuroReport 13, 1825-1828 (2002).

52. AlonsoAdel, C., Mederlyova, A., Novak, M., Grundke-Iqbal, I. \& Iqbal, K. Promotion of hyperphosphorylation by frontotemporal dementia tau mutations. J. Biol. Chem. 279, 34873-34881 (2004).

53. Sen, T., Saha, P., Jiang, T. \& Sen, N. Sulfhydration of AKT triggers Tau-phosphorylation by activating glycogen synthase kinase $3 \beta$ in Alzheimer's disease. Proc. Natl. Acad. Sci. USA. 117, 4418-4427 (2020).

54. Atagi, Y. et al. Apolipoprotein E is a ligand for triggering receptor expressed on myeloid cells 2 (TREM2). J. Biol. Chem. 290, 26043-26050 (2015).

55. Fitz, N. F. et al. Trem 2 deficiency differentially affects phenotype and transcriptome of human APOE3 and APOE4 mice. Mol. Neurodegener. 15, 41 (2020).

56. Syed, Y. Y. \& Deeks, E. $\left[{ }^{18} \mathrm{~F}\right]$ Florbetaben: a review in $\beta$-amyloid PET imaging in cognitive impairment. CNS Drugs 29, 605-613 (2015).

57. Nong, R. Y. et al. Corrigendum: Solid-phase proximity ligation assays for individual or parallel protein analyses with readout via real-time PCR or sequencing. Nat. Protoc. 10, 643 (2015).

\section{Acknowledgements}

This study was supported by a grant from the Korea Health Technology R\&D Project and Ministry of Health \& Welfare, Republic of Korea (\#HI18C1254). This work was partly supported by an institute of Information \& Communication Technology Planning \& Evaluation (IITP) grant funded by the Korean government (MIST) (No.2020-0-01373, Artificial Intelligence Graduate School Programing [Hanyang University]) and the research fund of Hanyang University (HY-202000000790011). We would like to express our sincere gratitude for the generous help of professor Masood Kamali-Moghaddam at Uppsala University about the procedure of SOLID phase PLA.

\section{Author contributions}

S.-H.P. and E.-H.L.: Methodology, formal analysis, and writing-original draft preparation. S.W.S.: Supervision and data curation. H.-J.K.: Formal data analysis, florbetaben PET reading. S.J.: Data curation. S.L.: Formal analysis. H.-H.P.: Investigation and laboratory data analysis. J.-H.L. and S.-H.K. (corresponding authors): Conceptualization, supervision, and project administration. So-H.P. and E.-H.L. contributed equally to this work.

\section{Competing interests}

The authors declare no competing interests.

\section{Additional information}

Correspondence and requests for materials should be addressed to S.-H.K. or J.-H.L.

Reprints and permissions information is available at www.nature.com/reprints.

Publisher's note Springer Nature remains neutral with regard to jurisdictional claims in published maps and institutional affiliations.

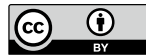

Open Access This article is licensed under a Creative Commons Attribution 4.0 International License, which permits use, sharing, adaptation, distribution and reproduction in any medium or format, as long as you give appropriate credit to the original author(s) and the source, provide a link to the Creative Commons licence, and indicate if changes were made. The images or other third party material in this article are included in the article's Creative Commons licence, unless indicated otherwise in a credit line to the material. If material is not included in the article's Creative Commons licence and your intended use is not permitted by statutory regulation or exceeds the permitted use, you will need to obtain permission directly from the copyright holder. To view a copy of this licence, visit http://creativecommons.org/licenses/by/4.0/.

(C) The Author(s) 2021 